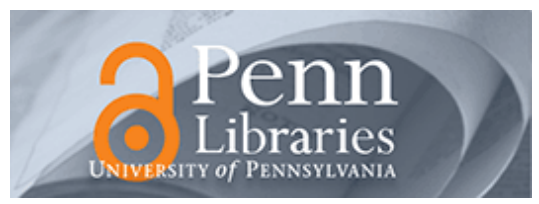

University of Pennsylvania

ScholarlyCommons

Operations, Information and Decisions Papers

Wharton Faculty Research

$1-1-2011$

\title{
Early Biometric Lag in the Prediction of Small for Gestational Age Neonates and Preeclampsia
}

\author{
Nadav Schwartz \\ University of Pennsylvania \\ Cara Pessel \\ Jaclyn Coletta \\ Abba M Krieger \\ Ilan E Timor-Tritsch
}

Follow this and additional works at: https://repository.upenn.edu/oid_papers

Part of the Medical Education Commons, Obstetrics and Gynecology Commons, and the Other Medicine and Health Sciences Commons

\section{Recommended Citation}

Schwartz, N., Pessel, C., Coletta, J., Krieger, A., \& Timor-Tritsch, I. (2011). Early Biometric Lag in the Prediction of Small for Gestational Age Neonates and Preeclampsia. Journal of ultrasound in medicine : official journal of the American Institute of Ultrasound in Medicine, 30 (1), 55-60. Retrieved from https://repository.upenn.edu/oid_papers/285

This paper is posted at ScholarlyCommons. https://repository.upenn.edu/oid_papers/285

For more information, please contact repository@pobox.upenn.edu. 


\title{
Early Biometric Lag in the Prediction of Small for Gestational Age Neonates and Preeclampsia
}

\author{
Abstract \\ OBJECTIVE: An early fetal growth lag may be a marker of future complications. We sought to determine \\ the utility of early biometric variables in predicting adverse pregnancy outcomes. \\ METHODS: In this retrospective cohort study, the crown-rump length at 11 to 14 weeks and the head \\ circumference, biparietal diameter, abdominal circumference, femur length, humerus length, transverse \\ cerebellar diameter, and estimated fetal weight at 18 to 24 weeks were converted to an estimated \\ gestational age using published regression formulas. Sonographic fetal growth (difference between each \\ biometric gestational age and the crown-rump length gestational age) minus expected fetal growth \\ (number of days elapsed between the two scans) yielded the biometric growth lag. These lags were \\ tested as predictors of small for gestational age (SGA) neonates ( $\leq 10$ th percentile) and preeclampsia.
}

RESULTS: A total of 245 patients were included. Thirty-two (13.1\%) delivered an SGA neonate, and 43 (17.6\%) had the composite outcome. The head circumference, biparietal diameter, abdominal circumference, and estimated fetal weight lags were identified as significant predictors of SGA neonates after adjusted analyses $(P<.05)$. The addition of either the estimated fetal weight or abdominal circumference lag to maternal characteristics alone significantly improved the performance of the predictive model, achieving areas under the curve of 0.72 and 0.74 , respectively. No significant association was found between the biometric lag variables and the development of preeclampsia.

CONCLUSIONS: Routinely available biometric data can be used to improve the prediction of adverse outcomes such as SGA. These biometric lags should be considered in efforts to develop screening algorithms for adverse outcomes.

\section{Keywords}

biometry, fetal growth restriction, intrauterine growth restriction, preeclampsia, sonography

\section{Disciplines}

Medical Education | Obstetrics and Gynecology | Other Medicine and Health Sciences 


\title{
Early Biometric Lag in the Prediction of Small for Gestational Age Neonates and Preeclampsia
}

\author{
Nadav Schwartz, MD, Cara Pessel, MD, Jaclyn Coletta, MD, Abba M. Krieger, PhD, Ilan E. Timor-Tritsch, MD
}

Supported in part by the American Institute of Ultrasound in Medicine Endowment for Education and Research
Received June 16, 2010, from the Department of Obstetrics and Gynecology, Maternal and Child Health Research Program, Hospital of the University of Pennsylvania, Philadelphia, Pennsylvania USA (N.S.); Department of Obstetrics and Gynecology, New York University Medical Center, New York, New York USA (N.S., C.P., J.C., I.E.T.-T.); and Department of Statistics, Wharton School of the University of Pennsylvania, Philadelphia, Pennsylvania USA (A.M.K.). Revision requested July 7, 2010. Revised manuscript accepted for publication July 22, 2010.

Address correspondence to Nadav Schwartz, MD, Department of Obstetrics and Gynecology, Division of Maternal-Fetal Medicine, Hospital of the University of Pennsylvania, 3400 Spruce St, 2000 Courtyard, Philadelphia, PA 19104 USA.

E-mail:schwartn@obgyn.upenn.edu

\section{Abbreviations}

$R O C$, receiver operator characteristic; SGA, small for gestational age
Objectives-An early fetal growth lag may be a marker of future complications. We sought to determine the utility of early biometric variables in predicting adverse pregnancy outcomes.

Methods - In this retrospective cohort study, the crown-rump length at 11 to 14 weeks and the head circumference, biparietal diameter, abdominal circumference, femur length, humerus length, transverse cerebellar diameter, and estimated fetal weight at 18 to 24 weeks were converted to an estimated gestational age using published regression formulas. Sonographic fetal growth (difference between each biometric gestational age and the crown-rump length gestational age) minus expected fetal growth (number of days elapsed between the two scans) yielded the biometric growth lag. These lags were tested as predictors of small for gestational age (SGA) neonates ( $\leq 10$ th percentile) and preeclampsia.

Results - A total of 245 patients were included. Thirty-two (13.1\%) delivered an SGA neonate, and $43(17.6 \%)$ had the composite outcome. The head circumference, biparietal diameter, abdominal circumference, and estimated fetal weight lags were identified as significant predictors of SGA neonates after adjusted analyses $(P<.05)$. The addition of either the estimated fetal weight or abdominal circumference lag to maternal characteristics alone significantly improved the performance of the predictive model, achieving areas under the curve of 0.72 and 0.74 , respectively. No significant association was found between the biometric lag variables and the development of preeclampsia.

Conclusions - Routinely available biometric data can be used to improve the prediction of adverse outcomes such as SGA. These biometric lags should be considered in efforts to develop screening algorithms for adverse outcomes.

Key Words-biometry; fetal growth restriction; intrauterine growth restriction; preeclampsia; sonography

E arly reliable predictors of placental dysfunction remain lacking in obstetric care. In reality, it is likely that no single test will achieve sufficient accuracy to be used in clinical practice as a stand-alone test in the prediction of pregnancy complications. Early identification of a pregnancy at risk of impaired fetal growth and an adverse outcome may allow for increased fetal surveillance to be implemented with the goal of preventing an adverse outcome. In addition, future medical advances may yield effective therapeutic options that, if used early in appropriately identified high-risk pregnancies, may decrease the incidence of adverse outcome. 
Although there is an abundance of evidence linking various maternal serum analytes and uterine artery Doppler measurements with adverse pregnancy outcomes, ${ }^{1-3}$ no single test has proved effective as a screening tool. Rather, recent evidence has shown that maternal risk factors, serum markers, and uterine artery Doppler studies can be combined in various ways to improve the overall prediction of adverse obstetric outcomes, such as fetal growth restriction and preeclampsia. ${ }^{4-6}$ Thus, the identification of additional early predictors of adverse outcomes may prove instrumental in the development of effective screening strategies.

It is biologically plausible that early lags in fetal biometry are a result of early placental dysfunction, leading to slowed fetal growth. It may be that in some cases, the growth lag persists and results in clinical growth restriction. In other cases, the growth lag may remain subclinical as continued placental growth and villous arborization are sufficient to support adequate fetal growth. However, the impact of impaired early placental development may still manifest as other obstetric complications such as preeclampsia. In fact, several studies have shown that a lag in biparietal diameter growth between the first and second trimesters is associated with adverse pregnancy outcomes, including small-for-gestational-age (SGA) neonates, low birth weight, preterm birth, and fetal death. ${ }^{7-9}$ Similarly, Fox et a $1{ }^{10}$ retrospectively examined a composite estimated fetal weight and found that pregnancies with an estimated fetal weight below the 25 th percentile for gestational age at 16 to 24 weeks were associated with increased rates of SGA neonates, low birth weight, indicated preterm birth, preeclampsia, and perinatal death. Thus, early biometric variables have the potential to serve as readily available predictors of adverse pregnancy outcomes.

We sought to expand on the existing evidence related to early biometry as a predictor of adverse outcomes by investigating whether other biometric parameters commonly recorded during the anatomic survey can be used to help identify pregnancies at risk for adverse outcomes.

\section{Materials and Methods}

The study population consisted of women who were enrolled in a prospective study investigating 3-dimensional placental sonography in early pregnancy. These women presented for nuchal translucency screening at 11 to 14 weeks and provided written informed consent. This protocol was approved by the Institutional Review Board. For this study, we identified women who underwent sonographic biometric assessment in our unit at both 11 to 14 and 18 to 24 weeks and for whom delivery outcome data were available.

This was the starting reference point for use in our calculation of interval fetal growth. The crown-rump length was collected from the first-trimester sonographic examination, and the biparietal diameter, head circumference, abdominal circumference, femur length, humerus length, and transverse cerebellar diameter were collected from the second-trimester anatomic survey. Each biometric variable was converted to an estimated gestational age by commonly used published regression formulas. ${ }^{11-15}$ For the purposes of our study, the gestational age based on the estimated fetal weight was calculated using the regression equation that includes the head circumference, biparietal diameter, abdominal circumference, and femur length because the combination of these 4 biometric parameters is commonly used to estimate the fetal weight on sonography (Table 1).

Interval fetal growth was calculated as the difference in the estimated gestational age (in days) between the two scans. We then calculated the biometric growth lag for each variable as the difference between the observed interval growth and the expected interval growth (ie, the number of calendar days elapsed between the first and second sonographic examinations). For example, if 8 weeks elapsed between the first-trimester scan and the second-trimester anatomic survey, then the expected growth would be 56 days. If the crown-rump length measurement estimated the gestational age to be 12 weeks and the biparietal diameter at the anatomic survey estimated the gestational age to be 19 weeks 2 days, then the observed biparietal diameter growth would be 7 weeks 2 days or 51 days. A biparietal diameter lag of -5 days (51-56) is the difference between

Table 1. Biometry Regression Formulas

\begin{tabular}{|c|c|}
\hline $\begin{array}{l}\text { Biometric } \\
\text { Variable }\end{array}$ & $\begin{array}{c}\text { Regression } \\
\text { Formula }\end{array}$ \\
\hline $\mathrm{CRL}^{11}$ & $\begin{array}{l}\operatorname{Ln}(G A)=1.684969+(0.315646 * C R L)- \\
\left(0.049306^{*} \mathrm{CRL}^{2}\right)+\left(0.004057^{\star} \mathrm{CRL}^{3}\right)- \\
\left(0.000120456{ }^{*} \mathrm{CRL}^{4}\right)\end{array}$ \\
\hline $\mathrm{HC}^{12}$ & $\mathrm{GA}=8.8+0.55^{*} \mathrm{HC}+0.0003 * \mathrm{HC}^{3}$ \\
\hline $\mathrm{BPD}^{13}$ & $\mathrm{GA}=9.54+1.482{ }^{*} \mathrm{BPD}+0.1676{ }^{*} \mathrm{BPD}^{2}$ \\
\hline $\mathrm{AC}^{13}$ & $\mathrm{GA}=8.14+0.753^{*} \mathrm{AC}+0.0036^{*} \mathrm{AC}^{2}$ \\
\hline $\mathrm{FL}^{13}$ & $\mathrm{GA}=10.35+2.46{ }^{*} \mathrm{FL}+0.17{ }^{*} \mathrm{FL}^{2}$ \\
\hline $\mathrm{EFW}^{13}$ & $\mathrm{GA}=10.85+0.06{ }^{*} \mathrm{HC} * \mathrm{FL}+0.67{ }^{*} \mathrm{BPD}+0.168{ }^{*} \mathrm{AC}$ \\
\hline $\mathrm{HL}^{14}$ & $\mathrm{GA}=9.6519438+0.26200391{ }^{*} \mathrm{HL}+0.0026105367{ }^{*} \mathrm{HL}^{2}$ \\
\hline $\mathrm{TCD}^{15}$ & $\mathrm{GA}=8.119+4.244{ }^{*} \mathrm{TCD}+1.1130 * \mathrm{TCD}^{2}-0.169 * \mathrm{TCD}^{3}$ \\
\hline
\end{tabular}

AC indicates abdominal circumference; BPD, biparietal diameter; CRL, crown-rump length; EFW, estimated fetal weight; FL, femur length; GA, gestational age; $\mathrm{HC}$, head circumference; $\mathrm{HL}$, humerus length; and TCD, transverse cerebellar diameter. 
the observed and expected growth. By defining an interval growth between two sonographic measurements, we avoided the potential variability and inaccuracy associated with the last menstrual period.

Outcome data were collected from the hospital medical record at time of delivery. Data collected included maternal age, parity, ethnicity, gestational age at delivery, birth weight, and the presence or absence of preeclampsia. Gestational age was based on first-trimester sonographic dating if a definite last menstrual period was not available or if there was a greater than 7-day discrepancy between menstrual dating and first-trimester sonographic biometric measurements.

The primary outcomes of the study were delivery of an SGA neonate and the development of preeclampsia. Small for gestational age was defined as a birth weight at or below the 10th percentile for gestational age using a national reference nomogram. ${ }^{16}$ A diagnosis of preeclampsia required systolic blood pressure elevations of greater than $140 \mathrm{~mm} \mathrm{Hg}$ or a diastolic blood pressure of greater than 90 $\mathrm{mm} \mathrm{Hg}$ and documentation of at least 1+ protein on a urine dipstick test or a 24-hour urine collection with greater than $300 \mathrm{mg}$ of protein.

Univariate analyses were first performed to identify potential associations between predictor variables and outcome variables. The Student $t$ test was used for continuous variables because the data were not particularly skewed. $\chi^{2}$ and Fisher exact tests were used for categorical variables. Parity was analyzed as a dichotomous variable (nulliparous or parous). Logistic regression was used to predict adverse outcomes, and receiver operator characteristic (ROC) curves were constructed for the biometric variables found to have significant associations with outcomes. Test performance characteristics were calculated for candidate cut points for each significant biometric variable. Finally, background variables were added to the logistic regression to adjust for potential confounders. Adjusted ROC curves were constructed, and the areas under the curve were recorded. These adjusted curves were compared using tests of equality as described by Cleves. ${ }^{17}$ Statistical analyses were performed using StataIC version 10.1 software (StataCorp, College Station, TX). $P<.05$ was considered statistically significant.

\section{Results}

We identified 245 patients who underwent first- and second-trimester sonography in our unit and for whom pregnancy outcome data were available. No major fetal malformations or intrauterine deaths were encountered.
The mean (SD) gestational age at delivery in the cohort was 39.1 (1.6) weeks, with 17 patients delivering before 37 weeks, for a preterm birth rate of $6.9 \%$. The mean birth weight in this cohort was 3287 (491) g, with 32 patients (13.1\%) delivering an SGA neonate and 12 (4.9\%) delivering a neonate weighing less than $2500 \mathrm{~g}$. Thirteen women $(5.3 \%)$ had a diagnosis of preeclampsia. Eighteen neonates $(7.3 \%)$ required admission to the neonatal intensive care unit.

Demographic data regarding maternal age, parity, and ethnicity are shown in Table 2 . No statistically significant difference was observed for the mean maternal age or ethnicity between those women who delivered an SGA neonate and those who delivered an appropriate-for-gestational-age neonate. There was, however, a statistically significant increase in the representation of nulliparous women among those who delivered an SGA neonate $(P=.04)$.

Fetuses who were eventually delivered SGA had a statistically significantly greater estimated fetal weight lag $(P=.007)$, head circumference lag $(P=.017)$, and abdominal circumference lag $(P=.001)$ during their secondtrimester anatomic survey (Table 3 ). In addition, there was a trend toward a greater biparietal diameter lag $(P=.055)$. Mean femur length, humerus length, and transverse cerebellar diameter lags were not significantly different between groups.

We then chose various lag cut points for each of the promising biometric lag variables (Table 4). As expected, there was a trade-off between the screen-positive rate, sensitivity, and specificity for each biometric variable. The use of a smaller lag to define a positive test result led to improved sensitivity in detecting a future SGA neonate but

Table 2. Demographic Characteristics

\begin{tabular}{|c|c|c|c|c|}
\hline Characteristic & $\begin{array}{c}\text { All } \\
(n=245)\end{array}$ & $\begin{array}{l}\text { Appropriate for } \\
\text { Gestational } \\
\text { Age }(n=211)\end{array}$ & $\begin{array}{l}\text { Small for } \\
\text { Gestational } \\
\text { Age }(n=32)\end{array}$ & $P$ \\
\hline $\begin{array}{c}\text { Maternal age, y, } \\
\text { mean (SD) }\end{array}$ & $34.0(4.4)$ & $34.1(4.4)$ & 33.6 (3.9) & $.56^{a}$ \\
\hline Parity, n (\%) & & & & $.04^{\mathrm{b}}$ \\
\hline Nulliparous & $142(58.4)$ & 118 (55.9) & $24(75)$ & \\
\hline Parity $\geq 1$ & $101(41.6)$ & 93 (44.1) & $8(25)$ & \\
\hline Ethnicity, n (\%) & & & & $.42^{c}$ \\
\hline White & $143(58.4)$ & 128 & 15 & \\
\hline Asian & $56(22.9)$ & 48 & 8 & \\
\hline Hispanic & $30(12.2)$ & 24 & 6 & \\
\hline Black & $15(6.1)$ & 12 & 3 & \\
\hline Other & $1(0.4)$ & 1 & 0 & \\
\hline
\end{tabular}

astudent $t$ test.

${ }^{\mathrm{b}} \chi^{2}$ test.

cFisher exact test. 
Table 3. Relationship Between Biometric Lags and Small for Gestational Age

\begin{tabular}{lcclll}
\hline $\begin{array}{l}\text { Sonographic } \\
\text { Variable }\end{array}$ & $\begin{array}{c}\text { Appropriate for } \\
\text { Gestational } \mathbf{A g e}\end{array}$ & $\begin{array}{c}\text { Small for } \\
\text { Gestational Age }\end{array}$ & $\mathbf{P b}^{\mathbf{a}}$ & $\mathbf{a O R}^{\mathbf{c}}$ & $\mathbf{P}^{\mathbf{c}}$ \\
\hline EFW lag & $-2.4(-2.8$ to -1.9$)$ & $-4.1(-5.0$ to -3.2$)$ & .007 & $0.84(0.75$ to 0.95$)$ & .005 \\
HC lag & $-2.3(-2.8$ to -1.7$)$ & $-4.1(-5.4$ to -2.8$)$ & .017 & $0.88(0.80$ to 0.97$)$ & .01 \\
BPD lag & $-0.1(-0.8$ to 0.6$)$ & $-2.0(-3.6$ to -0.4$)$ & .055 & $0.92(0.86$ to 0.995$)$ & .037 \\
AC lag & $0.9(0.3$ to 1.5$)$ & $-1.9(-3.2$ to -0.6$)$ & .001 & $0.86(0.78$ to 0.95$)$ \\
FL lag & $-0.2(-0.8$ to 0.5$)$ & $-1.4(-2.8$ to 0.0$)$ & .18 & & .002 \\
HL lag $(n=243)$ & $2.0(1.3$ to 2.8$)$ & $2.3(0.7$ to 3.9$)$ & .8 & & \\
TCD lag $(n=175)$ & $-0.6(-1.5$ to 0.4$)$ & $-2.1(-3.7$ to -0.5$)$ & .2 & \\
\hline
\end{tabular}

AC indicates abdominal circumference; aOR, adjusted odds ratio; BPD, biparietal diameter; EFW, estimated fetal weight; FL, femur length; $\mathrm{HC}$, head circumference; $\mathrm{HL}$, humerus length; and TCD, transverse cerebellar diameter.

aMean biometric lag in days (95\% confidence interval).

bStudent $t$ test.

cAdjusted for maternal age, ethnicity, and parity.

at the expense of a higher screen-positive rate and lower specificity. Thus, although the sensitivities ranged from $21 \%$ to $78 \%$, the higher sensitivities seen with the estimated fetal weight and head circumference lags were associated with screen positive rates of greater than $40 \%$ and specificities of no more than $61 \%$. It can be argued that the optimal trade-off from among these variables comes with the use of an abdominal circumference lag of -3 days, which detected almost $45 \%$ of SGA neonates with just more than one-fourth of patients screening positive. However, even with this definition, only 1 of 5 screen-positive women delivered an SGA neonate when the abdominal circumference lag was used as a stand-alone test.

In the multivariable models, the estimated fetal weight, head circumference, biparietal diameter, and abdominal circumference lags remained significantly associated with SGA neonates even after adjusting for maternal characteristics (age, ethnicity, and parity; Table 3).
In Figure 1, the ROC curve for maternal characteristics alone is plotted alongside the curves obtained when each biometric lag variable was included in the adjusted model. The addition of the estimated fetal weight lag $(P=$ $.037)$ or abdominal circumference lag $(P=.016)$ to maternal characteristics resulted in a statistically significant improvement in the model's performance. On the other hand, the addition of the biparietal diameter lag $(P=.13)$ or head circumference lag $(P=.12)$ to maternal characteristics did not result in a statistically significant improvement in testing performance compared with maternal characteristics alone.

There were no significant differences in mean lags for any of the biometric variables between pregnancies that developed preeclampsia and those that did not (Table 5).

Table 4. Test Characteristics for Biometric Lags as Predictors of Small for Gestational Age

\begin{tabular}{|c|c|c|c|c|c|c|}
\hline $\begin{array}{l}\text { Sonographic } \\
\text { Variable }\end{array}$ & $\begin{array}{c}\text { Cut } \\
\text { Point, d }\end{array}$ & $\begin{array}{l}\text { Screen-Positive } \\
\text { Rate, \% }\end{array}$ & $\begin{array}{l}\text { Sensitivity, \% } \\
(95 \% \mathrm{Cl})\end{array}$ & $\begin{array}{l}\text { Specificity, \% } \\
(95 \% \mathrm{Cl})\end{array}$ & $\begin{array}{l}\text { Positive Predictive } \\
\text { Value, \% }(95 \% \mathrm{Cl})\end{array}$ & $\begin{array}{l}\text { Negative Predictive } \\
\text { Value, \% }(95 \% \mathrm{Cl})\end{array}$ \\
\hline \multirow[t]{3}{*}{ EFW lag } & -3 & 52.2 & 78.1 (59.6 to 90.1) & 51.6 (44.7 to 58.5) & 19.5 (13.3 to 27.7) & 94.0 (87.6 to 97.4$)$ \\
\hline & -4 & 42.4 & 65.6 (46.8 to 80.8$)$ & 61.0 (54.1 to 67.6) & 20.2 (13.2 to 29.4$)$ & 92.2 (86.1 to 95.8$)$ \\
\hline & -5 & 29.8 & 37.5 (21.7 to 56.3) & 71.4 (64.7 to 77.2 ) & 16.4 (9.1 to 27.3 ) & 88.4 (82.4 to 92.6$)$ \\
\hline \multirow[t]{3}{*}{ AClag } & -3 & 27.8 & 43.8 (26.8 to 62.1$)$ & 74.6 (68.2 to 80.2) & 20.6 (12.1 to 32.5) & 89.8 (84.2 to 93.7) \\
\hline & -4 & 20.0 & 28.1 (14.4 to 47.0$)$ & 81.2 (75.2 to 86.1) & $18.4(9.2$ to 32.5$)$ & 88.3 (82.7 to 92.3 ) \\
\hline & -5 & 14.3 & 21.9 (9.9 to 40.4$)$ & 86.9 (81.4 to 90.9) & 20.0 (9.1 to 37.5$)$ & 80 (62.5 to 90.9$)$ \\
\hline \multirow[t]{3}{*}{$\mathrm{HC}$ lag } & -3 & 49.4 & 68.8 (49.9 to 83.3) & 53.5 (46.6 to 60.3 ) & 18.2 (12 to 26.5 ) & 91.9 (85.3 to 95.8) \\
\hline & -4 & 41.6 & 59.4 (40.8 to 75.8$)$ & $61.0(54.1$ to 67.6$)$ & 18.6 (11.9 to 27.8$)$ & 90.9 (84.7 to 94.9$)$ \\
\hline & -5 & 33.5 & 46.9 (29.5 to 65$)$ & 68.5 (61.8 to 74.6$)$ & 18.3 (10.9 to 28.7) & 89.6 (83.6 to 93.6) \\
\hline \multirow[t]{3}{*}{ BPD lag } & -3 & 35.1 & 53.1 (35.0 to 70.5$)$ & 67.6 (60.8 to 73.7$)$ & 19.8 (12.3 to 30.0) & 90.6 (84.6 to 94.4$)$ \\
\hline & -4 & 28.6 & 40.6 (24.2 to 59.2) & 73.2 (66.7 to 78.9$)$ & 18.6 (10.6 to 30.0$)$ & 89.1 (83.3 to 93.2) \\
\hline & -5 & 20.8 & 25.0 (12.1 to 43.8$)$ & 79.8 (73.7 to 84.9) & 15.7 (7.5 to 29.1$)$ & 87.6 (82 to 91.8 ) \\
\hline
\end{tabular}

$\mathrm{AC}$ indicates abdominal circumference; $\mathrm{BPD}$, biparietal diameter; $\mathrm{Cl}$, confidence interval; $\mathrm{EFW}$, estimated fetal weight; and $\mathrm{HC}$, head circumference. 


\section{Discussion}

This study confirms the presence of early biometric lags in fetuses destined to be delivered SGA. Although the test characteristics of any single biometric lag variable are suboptimal for use as a stand-alone screening test, the addition of biometric lag variables to maternal characteristics improves overall prediction. In fact, incorporating the estimated fetal weight or abdominal circumference lag in the screening models for predicting SGA neonates led to statistically significant improvements in the ROCs of the model.

Many obstetric complications first become clinically apparent in the later stages of pregnancy. However, it is generally believed that many placenta-related complications such as fetal growth restriction and preeclampsia are related to poor trophoblastic invasion and placental development in early pregnancy. Therefore, it is biologically plausible that subtle signs of early placental inefficiency or dysfunction may serve as markers for future clinical disease.

Figure 1. Receiver operating characteristic curves using each of the significant biometric lag variables adjusted for maternal characteristics (age, ethnicity, and parity) in the prediction of small for gestational age neonates. The receiver operating characteristic curve using maternal characteristics alone is also displayed. Area under the curve values are shown, along with the $P$ values for the comparison of each adjusted biometric lag model with the model using only maternal characteristics. The receiver operating characteristic curves for the estimated fetal weight lag and abdominal circumference lags are significantly more predictive than the curve for maternal characteristics alone. AC indicates abdominal circumference; BPD, biparietal diameter; EFW, estimated fetal weight; and $\mathrm{HC}$, head circumference.

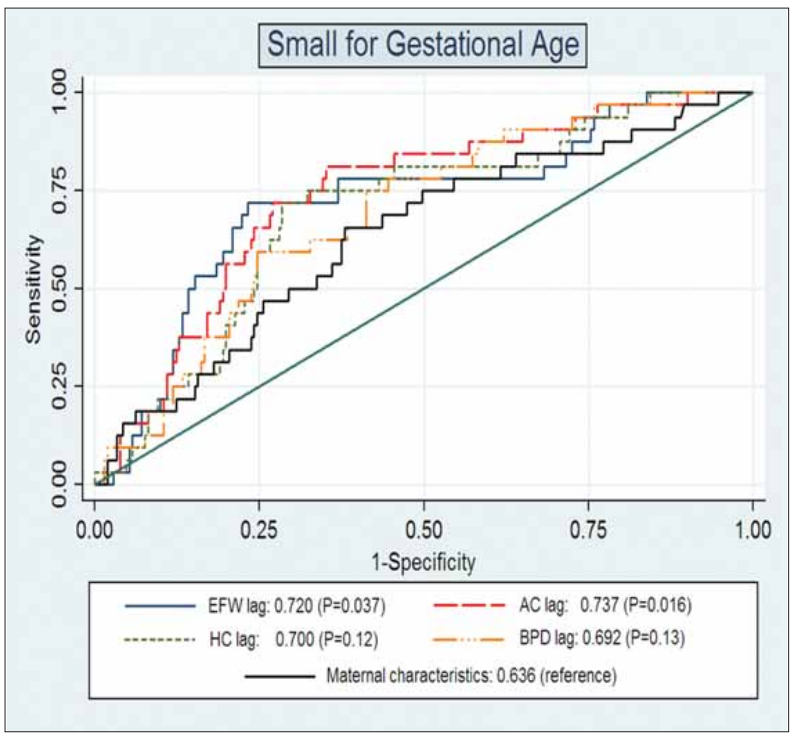

Although fetal growth restriction has been associated with both short- and long-term adverse outcomes, ${ }^{18,19}$ studies show that approximately one-third of growthrestricted fetuses remain undetected before delivery. ${ }^{20}$ Although the greater fetal growth velocity in the late second and early third trimesters may allow for the emergence of clinically apparent growth restriction, smaller deviations from optimal growth in the first half of pregnancy may serve as early markers of future disease. Such early detection may lend itself to increased antenatal detection of fetal growth restriction and may allow for increased fetal surveillance to decrease the incidence of adverse outcomes such as fetal death. Furthermore, the identification of early predictors of other adverse outcomes such as preeclampsia may also allow for a more targeted approach to the investigation of candidate interventions being studied as potential therapies for these disease states.

Several studies have focused on biparietal diameter growth as an early predictor of outcomes. ${ }^{7-9}$ In one large retrospective cohort, Pedersen et a ${ }^{8}$ used the biparietal diameter growth rate from the first to second trimester as a predictor of adverse outcomes. Although their results indicated that biparietal diameter growth rates below the 2.5th percentile were associated with an increased risk of perinatal death and intrauterine growth restriction, they limited their analysis to the biparietal diameter and did not investigate other biometric parameters as potential predictors. The lack of a convincing association between the biparietal diameter lag and outcome in our study may have been due to the small size of our cohort, which limited our ability to detect a statistically significant difference. Nevertheless, the significant associations between both estimated fetal weight and abdominal circumference lags and SGA neonates in our small cohort indicate that other biometric variables may be even more predictive than the biparietal diameter lag.

Table 5. Relationship Between Biometric Lags and Preeclampsia

\begin{tabular}{lccc}
\hline $\begin{array}{l}\text { Sonographic } \\
\text { Variable }\end{array}$ & No Preeclampsia $^{\mathbf{a}}$ & Preeclampsia $^{\mathbf{a}}$ & $\boldsymbol{P}^{\mathbf{b}}$ \\
\hline EFW lag & $-2.6(-3.1$ to -2.2$)$ & $-2.3(-4.2$ to -4.6$)$ & .8 \\
HC lag & $-3.7(-1.0$ to 0.3$)$ & $0(-3.6$ to 3.6$)$ & .8 \\
BPD lag & $-2.5(-3.0$ to -2.0$)$ & $-3.2(-5.1$ to -1.2$)$ & .6 \\
AC lag & $0.4(-0.2$ to 1.0$)$ & $2.4(-0.6$ to 5.4$)$ & .1 \\
FL lag & $-0.4(-1.0$ to 0.2$)$ & $-0.2(-3.6$ to 3.3$)$ & .9 \\
HL lag $(n=243)$ & $2.0(+1.3$ to 2.6$)$ & $3.7(-1.4$ to 8.9$)$ & .2 \\
TCD lag $(n=175)$ & $-0.8(-1.7$ to 0.1$)$ & $-0.4(-3.9$ to 3.1$)$ & .9 \\
\hline
\end{tabular}

$\mathrm{AC}$ indicates abdominal circumference; BPD, biparietal diameter; EFW, estimated fetal weight; FL, femur length; HC, head circumference; $\mathrm{HL}$, humerus length; and TCD, transverse cerebellar diameter. ${ }^{a}$ Mean biometric lag in days ( $95 \%$ confidence interval).

bStudent $t$ test. 
In addition, because the abdominal circumference also plays a significant role in the estimated fetal weight formula, lags in abdominal circumference and estimated fetal weight may be early manifestations of "brain sparing," in which preferential shunting of blood to the brain leads to slower growth of the abdominal circumference.

The relatively small size of this study cohort limited our ability to expand on the number of risk factors included in our prediction models. For example, a larger cohort would have allowed us to analyze additional variables such as the body mass index, prior pregnancy history, and medical comorbidities to develop a clinically effective screening tool. However, although such a research goal would certainly be an important one, it would require a very large cohort with an extensive database of historical and clinical information to achieve. Thus, such extensive analyses were outside the scope of our study. Rather, our results argue that biometric lags have the potential to serve as biologically plausible and easily obtainable variables that have significant associations with SGA neonates. To this end, this study supports this hypothesis by showing the significant improvement in testing characteristics when adding biometric lags, such as the estimated fetal weight and abdominal circumference lags, to maternal age and ethnicity. It remains to be seen whether these findings remain significant when more robust prediction models are used.

Our analyses did not reveal a significant association between early biometric lags and preeclampsia. Although this may also be due to the limited size of our cohort, these results are in agreement with those of Pedersen et al, ${ }^{8}$ who reported no association between early biparietal diameter growth rates and preeclampsia.

Although many pregnancy complications are believed to be manifestations of placental dysfunction, the exact mechanisms leading to the various adverse outcomes are poorly understood. To date, no single biomarker has been identified as an accurate predictor of outcomes. However, recent efforts have shown that readily available demographic and clinical variables can improve the performance of novel predictors. Our results support the consideration of early biometric lags in research efforts seeking to develop a clinically reliable screening approach to predict adverse pregnancy outcomes such as SGA.

\section{References}

1. Gagnon A, Wilson RD, Audibert F, et al. Obstetrical complications associated with abnormal maternal serum markers analytes. J Obstet Gynaecol Can 2008; 30:918-949.
2. Tjoa ML, Oudejans CB, van VugtJM, Blankenstein MA, van Wijk II. Markers for presymptomatic prediction of preeclampsia and intrauterinegrowth restriction. Hypertens Pregnancy 2004; 23:171-189.

3. Cnossen JS, Morris RK, ter Riet G, et al. Use of uterine artery Doppler ultrasonography to predict pre-eclampsia and intrauterine growth restriction: a systematic reviewand bivariable meta-analysis. CMAJ2008;178:701-711.

4. Poon LC, Akolekar R, Lachmann R, Beta J, Nicolaides KH. Hypertensive disorders in pregnancy: screening by biophysical and biochemical markers at $11-$ 13 weeks. Ultrasound Obstet Gynecol 2010;35:662-670.

5. Poon LC, Stratieva V, Piras S, Piri S, Nicolaides KH. Hypertensive disorders in pregnancy: combined screening by uterine artery Doppler, blood pressure and serum PAPP-Aat 11-13 weeks. Prenat Diagn 2010; 30:216-223.

6. PilalisA,SoukaAP,AntsaklisP,etal.Screeningforpre-eclampsiaandfetal growth restriction by uterine artery Doppler and PAPP-A at 11-14 weeks' gestation. Ultrasound Obstet Gynecol2007;29:135-140.

7. PedersenNG,FiguerasF,Wojdemann KR, Tabor A, GardosiJ.Earlyfetal size and growth as predictors of adverse outcome. Obstet Gynecol 2008; 112:765-771.

8. PedersenNG,Wojdemann KR,ScheikeT,Tabor A.Fetal growth between the firstandsecond trimesters and therisk ofadversepregnancyoutcome.Ultrasound Obstet Gynecol 2008; 32:147-154

9. NaklingJ,BackeB.Adverse obstetricoutcomein fetuses that are smallerthan expected at second trimester routine ultrasound examination. Acta Obstet Gynecol Scand 2002; 81:846-851.

10. FoxNS,HuangM,ChasenST.Second-trimesterfetal growthandtherisk of poor obstetric and neonatal outcomes. Ultrasound Obstet Gynecol 2008;32:61-65.

11. Hadlock FP,Shah YP,KanonDJ,LindseyJV.Fetal crown-rumplength: reevaluation of relation to menstrual age (5-18 weeks) with high-resolution real-time US. Radiology 1992; 182:501-505.

12. Hadlock FP, Deter RL, Harrist RB, Park SK. Fetal head circumference: relation to menstrual age. AJRAmJ Roentgenol 1982; 138:649-653.

13. Hadlock FP, Deter RL, Harrist RB, Park SK. Estimating fetal age: computerassisted analysis of multiple fetal growth parameters. Radiology 1984; 152:497501.

14. JeantyP,RodeschF,DelbekeD,DumontJE.Estimation ofgestational agefrom measurements of fetallong bones.J Ultrasound Med 1984; 3:75-79.

15. Chavez MR, Ananth CV, Smulian JC, Yeo L, Oyelese Y, Vintzileos AM. Fetal transcerebellar diameter measurement with particular emphasis in the third trimester: a reliable predictor of gestational age. Am J Obstet Gynecol 2004; 191:979-984.

16. Alexander GR, Himes JH, Kaufman RB, Mor J,Kogan M.A United States national reference for fetal growth. Obstet Gynecol 1996; 87:163-168.

17. Cleves M. From the help desk: comparing areas under receiver operating characteristiccurves from two or more probitorlogitmodels.Stata 2002;2:301-313.

18. van Wassenaer A. Neurodevelopmental consequences of being born SGA.Pediatr Endocrinol Rev 2005; 2:372-377.

19. PallottoEK,KilbrideHW.Perinatal outcomeandlaterimplications ofintrauterine growth restriction. Clin Obstet Gynecol 2006; 49:257-269.

20. American Congress of Obstetricians and Gynecologists. Practice bulletin No. 12: intrauterine growth restriction. American Congress of Obstetricians and Gynecologists website. http://www.acog.org/publications/educational_ bulletins/pb012.cfm. Accessed May 1,2010. 